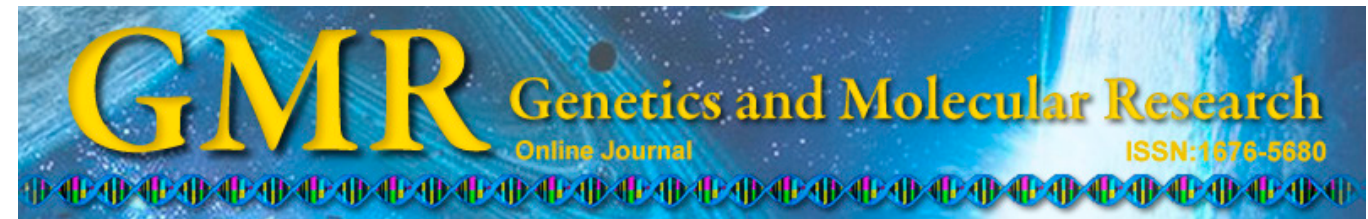

\title{
Analysis of alterations to the transcriptome of Loquat (Eriobotrya japonica Lindl.) under low temperature stress via de novo sequencing
}

\author{
R.G. Gong ${ }^{1,2,3}$, J. Lai ${ }^{1}$, W. Yang ${ }^{1,3}$, M.A. Liao ${ }^{1}$, Z.H. Wang ${ }^{1}$ and G.L. Liang ${ }^{2}$ \\ ${ }^{1}$ College of Horticulture, Sichuan Agricultural University, Ya' an, China \\ ${ }^{2}$ College of Horticulture and Landscape Architecture, Southwest University, \\ Chongqing, China \\ ${ }^{3}$ Longquanyi District Fruit Research Institute, Chengdu, China \\ Corresponding authors: R.G. Gong / G.L. Liang \\ E-mail: gongronggao2014@163.com / liangguolu2014@163.com
}

Genet. Mol. Res. 14 (3): 9423-9436 (2015)

Received October 24, 2014

Accepted May 8, 2015

Published August 14, 2015

DOI http://dx.doi.org/10.4238/2015.August.14.6

\begin{abstract}
Loquat (Eriobotrya japonica Lindl.), which originates from the cooler hill regions of southwestern China, is a typical subtropical evergreen tree. Loquat is one of the most important economic crops in China, but the available genomic information is very limited. Here, we present the first deep transcriptomic analysis of loquat. De novo assembly generated 116,723 contigs and 64,814 unigenes using Illumina sequencing technology. A total of 45,739 unigenes were annotated by $\mathrm{Nr}$, GO, and COG datasets. In addition, we analyzed the gene expression profiles of loquat fruit under low temperature stress and 4017 differential expressed genes were identified. We found that the unigenes involved in the brassinosteroid biosynthesis and phosphatidylinositol signaling systems were upregulated, indicating that they have an important role in the resistance of plants to low temperature. Our results provide an invaluable resource for identification of specific genes and proteins involved in loquat development and response to low temperatures.
\end{abstract}

Key words: Loquat; Illumina sequencing; Low temperature stress; Transcriptome 


\section{INTRODUCTION}

Loquat (Eriobotrya japonica Lindl. Rosaceae, Maloideae) is a typical subtropical, evergreen, fruit-bearing tree and most authors believe that it originated in the cooler hill regions of southwestern China (Campbell and Malo, 1986; Morton, 1987; Zhang et al., 1990, 1993). Loquat has been cultivated for more than 2000 years and the acreage has increased tremendously in the last few decades (Lin, 2007; Mortazavi et al., 2008). Sichuan is the principal province of loquat production in southwest China, with an acreage of over 61,334 ha, which achieved a yield of more than 360,000 tons with a total value of 2.88 billion RMB in 2008 (Jiang et al., 2001).

Loquat fruit ripens in spring and early summer, and the ripe fruit is round or oval in shape, from white to deep orange or salmon in color, with a thin, tough skin, and soft, juicy flesh (Lin et al., 1999). There are several biotic and abiotic constraints that limit plant production and quality; in particular loquat fruit is sensitive to chilling. Chilling injury causes a loss of flavor and taste, flesh woodiness, decreased juiciness, and, internal browning (Lin et al., 1999; Zheng et al., 2000; Ding et al., 2002; Cai et al., 2006a). Currently, most reports have focused on long-term storage of the fruit at low temperatures. Previous studies have indicated that low temperature inhibits fruit respiration and ethylene production (Ding et al., 1998), and it has been demonstrated that the quality of "Luoyangqing" loquat fruit stored at $5{ }^{\circ} \mathrm{C}$ is better than the quality of fruit stored at $0^{\circ} \mathrm{C}$ (Cai et al., 2006a,b). Chilling injury occurs in loquat fruit when they are stored at temperatures lower than $5^{\circ} \mathrm{C}$, and fruit stored at $0^{\circ} \mathrm{C}$ exhibits severe symptoms of lignification and tissue browning, and a decrease in the percentage of juice (Cai et al., 2006c). Wang et al. (2010) identified five components of the ethylene signal transduction pathway from ripening loquat fruit and found that the EjETR1 and EjEIL1 genes may be involved in chilling injury development. However, the expression profiles of these genes under low temperature stress are unknown.

In comparison to other fruit trees, very limited genomic information is available for loquat. Various genomic technologies have facilitated the identification and characterization of special genes and proteins in several plants (Varshney et al., 2005, 2006; Krost et al., 2012), and over the past several years, next-generation sequencing technology has emerged as a cutting edge approach, providing an opportunity to generate high throughput transcriptomic data efficiently, promptly and at a lower cost for non-model organisms. Next-generation sequencing technologies include the development of the Roche/454 GS FLX System, the Illumina Genome Analyzer/HiSeq systems, and the ABI SOLiD System. For example, Illumina sequencing technology is able to trace total RNA from an established linear amplification sequence library, and generate over one billion bases of high-quality DNA sequence per run at less than $1 \%$ of the cost of capillary-based methods (Huang and Marth, 2008). Furthermore, next generation sequencing technology has also significantly accelerated and improved the sensitivity of gene-expression profiling and is expected to boost collaborative and comparative genomics studies (Rosenkranz et al., 2008). Despite being used in several crop species, Illumina sequencing methods have not yet been applied to loquat research. Here, we present a de novo assembly approach for the transcriptome of loquat.

In the present study, Illumina technology was used to characterize the transcriptome of loquat. To our knowledge, this is the first report to provide complete transcriptome of loquat, and our results contribute to future genetic and genomic studies on loquat and provide a 
foundation for identifying specific genes and proteins involved in loquat development. Moreover, we analyzed the gene expression profiles of loquat fruit under low temperature stress. This dataset will provide an invaluable resource for the identification of loquat fruit genes involved in low temperature stress. We believe that knowledge of stress genes is very important for the continued development of rational breeding and transgenic strategies to improve low temperature stress resistance in crops.

\section{MATERIAL AND METHODS}

\section{Plant material and low temperature stress}

Loquat (E. japonica Lindl. cv. Dawuxing) fruit was picked in the Longquanyi district in Chengdu, Sichuan Province, China, and transported to the laboratory on the day of collection. For the low temperature experiment, the fruit was stored at $3^{\circ} \mathrm{C}$ for $12 \mathrm{~h}$ in a controlled culture room. The fruit was uniform in size and maturity and without mechanical damage, and a completely randomized design was used in the experiment.

\section{Material handling for qRT-PCR}

Thirty fruit spurs taken from the crown of young fruit were used in the experiment. Of these, 10 were placed in an artificial climate box at $12^{\circ} \mathrm{C}$ as a control, 10 were placed at a processing low temperature stress of $6^{\circ} \mathrm{C}$ as a treatment, and 10 were placed at a processing low temperature stress of $3^{\circ} \mathrm{C}$ as a treatment, for $12 \mathrm{~h}$.

\section{RNA extraction and quality control}

Total RNA was extracted from loquat fruit using the SV total RNA isolation system (Promega) according to the manufacturer protocol. The quality and quantity of total RNA samples were assessed as previously described (Garg et al., 2010). Only RNA samples with a A260/A280 ratio in the range 1.9-2.1 and a A260/A230 ratio in the range 2.0-2.5 were used in the analysis. The integrity of the RNA samples was verified using the Agilent 2100 Bioanalyzer. Samples with an RIN (RNA integrity number) value ranging from 8.6-10.0 were utilized for further analysis.

\section{Illumina cDNA library construction and sequencing}

Two cDNA libraries (loquat fruit without or with low temperature stress treatment) were generated using Illumina's kit following the manufacturer protocol. Total RNA was treated with DNase I prior to library construction and poly-(A) mRNA was first purified with magnetic oligo(dT) beads. To avoid priming bias, mRNA was fragmented into small pieces by treating with divalent cations and heat. Following cleavage, the RNA fragments were transcribed into first strand cDNA using reverse transcriptase and random hexamer-primers, followed by second-strand cDNA synthesis using DNA polymerase I and RNase H. Doublestranded cDNA was further end-repaired using T4 DNA polymerase. These products were selectively enriched and the cDNA fragments were amplified using PCR to create the final 
cDNA library. After validation with an Agilent 2100 Bioanalyzer and ABI StepOne Plus RealTime PCR System, the cDNA library was sequenced using an Illumina HiSeq ${ }^{\mathrm{TM}} 2000$ sequencing platform.

\section{Sequence data analysis and de novo assembly}

The raw reads were assembled separately and adapter sequences, empty reads, and low quality sequences (reads with unknown sequences, ' $N>5 \%$ '), and reads with more than $10 \% \mathrm{Q} \leq 20$ bases were removed.

De novo assembly of the clean reads was performed using Trinity, which recovers more full length transcripts across a broad range of expression levels, with sensitivity similar to methods that rely on genome alignments (Grabherr et al., 2011). The best assembly was achieved with $\mathrm{K}=25$, which was chosen for de Bruijn graph construction.

\section{Sequence annotation}

To analyze the most descriptive annotation for each sequence, unigenes were annotated based on a set of sequential BLAST searches (Altschul et al., 1997). The unigenes were compared against the National Center for Biotechnology Information (NCBI) non-redundant protein $(\mathrm{Nr})$ and Swiss-Prot protein databases using the BLASTx algorithm with an E-value of less than $10^{-5}$. Based on the Nr BLAST results, gene ontology terms (GO; http://www.geneontology.org) annotation of the unigenes was obtained using the Blast2GO software (Conesa et al., 2005). The WEGO package was used for GO functional classification of all unigenes and to plot the distribution of gene functions. The unigene sequences were also aligned to the clusters of orthologous groups $(\mathrm{COG})$ database to predict their functions.

\section{Evaluation of differentially expressed genes under low temperature stress}

To calculate the expression of unigenes, the FPKM (fragments per KB per million fragments) algorithm was used (Chen and Cai, 1988). This method can eliminate the influence of gene length on calculations of gene expression, and the result can be directly used to compare different gene expression patterns among samples. Statistical analysis of the different cDNA libraries was performed to compare gene expression under low temperature stress using the method described by Audic and Claverie (1997). A false discovery rate of $<0.001$ was used as the threshold to judge the significance of the gene expression difference, which determined the threshold of the P value in multiple tests and analysis. For pathway enrichment analysis, all differentially expressed genes were searched against the Kyoto encyclopedia of genes and genomes (KEGG) database and searched for significantly enriched KEGG terms compared to the genome background.

\section{qRT-PCR}

For qRT-PCR, approximately $3 \mathrm{mg}$ of total RNA was reverse transcribed using SuperScript II reverse transcriptase (Invitrogen) in a volume of $100 \mathrm{~mL}$ to obtain cDNA. We carried out qRT-PCR in a total volume of $25 \mathrm{~mL}$ containing $2 \mathrm{~mL}$ of the reverse-transcribed product, 
$0.25 \mathrm{mM}$ gene-specific primers, and 12.5 mL SYBR Green Master Mix (Applied Biosystems) on a BIO-RAD CFX Connect Real-Time PCR System according to the manufacturer protocol. Primer pairs for qRT-PCR analysis are listed in Table 1. The measurements were obtained using the relative quantification method (Livak and Schmittgen, 2001).

\begin{tabular}{|c|c|c|c|}
\hline SN & Gene & Sequence $5^{\prime} \rightarrow 3^{\prime}$ & PCR efficiency \\
\hline 1 & ACTIN & $\begin{array}{l}\text { F: GTGCTTTCCCTCTATG CCAGTG } \\
\text { R: GAATAGCCACGCTCAGTCAAAA }\end{array}$ & $79.1 \%$ \\
\hline 2 & 25620 & $\begin{array}{l}\text { F: TGATCTGCTTGGATGGGTTTTG } \\
\text { R: TGCGTTAGGGCAGCTTGGTA }\end{array}$ & $96.0 \%$ \\
\hline 3 & C18135 & $\begin{array}{l}\text { F: CTCTTACTCCCAAGGCGACCA } \\
\text { R: CCACCTCTTTCCTCAAACTCCAA }\end{array}$ & $87.1 \%$ \\
\hline
\end{tabular}

\section{RESULTS}

\section{Sequence analysis and assembly}

To obtain a landscape of the loquat transcriptome, a cDNA sample, which was extracted from the pulp part of young loquat fruit (60 days after flowering), was sequenced using the Illumina sequencing platform. We generated a total of 30,911,414 raw reads. After high stringent quality assessment and data cleaning, 26,024,270 clean reads with 97.47\% Q20 bases (those with a base quality greater than 20 ) were selected as high quality reads for further analysis. The average GC content of the transcriptome was $48.94 \%$, ranging from $20.3-$ $70.71 \%$. An overview of the sequencing is presented in Table 2.

Table 2. Summary of Illumina transcriptome sequencing for loquat fruit.
\begin{tabular}{lccccc}
\hline Sample & Total raw reads & Total clean reads & Q20 percentage & N percentage & GC percentage \\
\hline Fruit (no stress) & $30,911,414$ & $26,024,270$ & $97.47 \%$ & $0.00 \%$ & $48.94 \%$ \\
Fruit (low temperature stress) & $30,896,730$ & $27,201,318$ & $97.39 \%$ & $0.00 \%$ & $48.47 \%$ \\
\hline
\end{tabular}

Using the Trinity de novo assembly program, next-generation short-read sequences were assembled into 116,723 contigs, the average contig length was 312 nucleotides (nt), and the N50 length was $487 \mathrm{bp}$ (Table 3). The contigs ranged from 200 to $7653 \mathrm{nt}$ in length (Figure 1A) and a total of 64,814 unigenes were assembled, with an N50 length of $797 \mathrm{bp}$ and a mean length of $541 \mathrm{nt}$ (Table 3). The length of unigenes ranged from 200 to $8895 \mathrm{nt}$, with a total length of 35,036,073 nt (Figure 1B). The length distributions of the contigs and unigenes are shown in Figure 1, revealing that 23.32 and $13.79 \%$ of reads are greater than $1000 \mathrm{nt}$, respectively.

\section{Sequence annotation}

Several complementary approaches were utilized to annotate the assembled sequences. The unigenes were first annotated using BLASTx against the NCBI Nr database. A total of $42,830 \mathrm{Nr}$ sequences were identified in the annotated genes and the E-value distribution showed that $42.5 \%$ of the sequences have strong homology (smaller than $1.0 \mathrm{E}^{-60}$ ), whereas 
$57.5 \%$ of the homolog sequences ranged between $1.0 \mathrm{E}^{-5}$ to $1.0 \mathrm{E}^{-60}$ (Figure $2 \mathrm{~A}$ ). For the similarity distribution, $29.7 \%$ of the hits had a similarity greater than $80 \%$, while $70.3 \%$ of the hits ranged from 19-80\% (Figure 2B). In Figure 2C, the species distribution showed 32.5\% of the sequences have high matches with sequences from Vitis vinifera, followed by Ricinus communis (18.4\%), Populus trichocarpa (16.4\%), Glycine max (11.3\%), Malus x domestica (4.1\%), Medicago truncatula (3.2\%), and Arabidopsis thaliana (1.1\%).

A

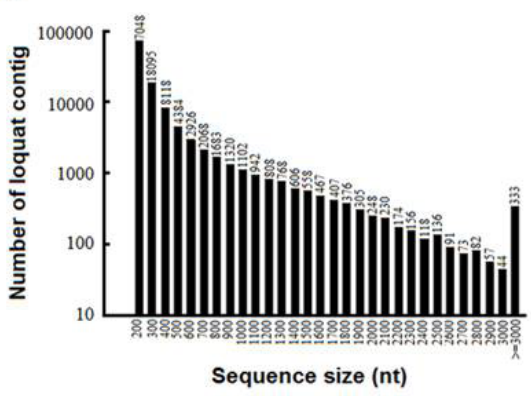

B

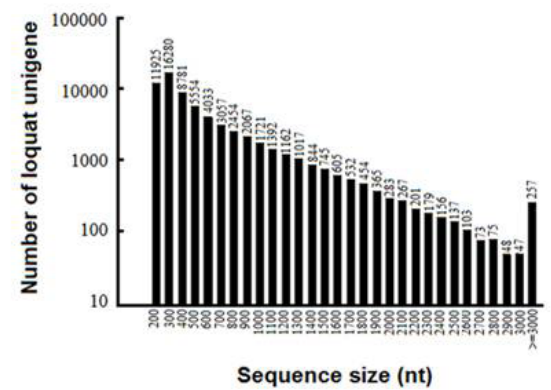

Figure 1. Length distribution of contigs (A) and unigenes (B) from the fully assembled loquat transcriptome.

Table 3. Summary statistics of assembled gene sequences.

\begin{tabular}{lcccc}
\hline & Total number & Total length (nt) & Mean length (nt) & N50 \\
\hline Contig & 116,723 & $36,387,214$ & 312 & 487 \\
Unigene & 64,814 & $35,036,073$ & 541 & 797 \\
\hline
\end{tabular}

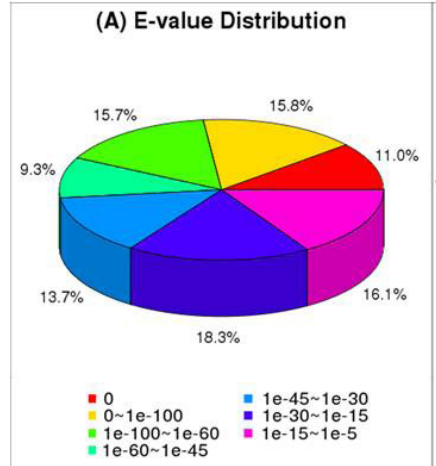

(B) Similarity Distribution

(C) Species Distribution

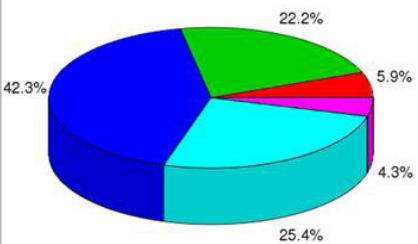

$\begin{array}{ll}-19 \% \sim 40 \% & =80 \% \sim 95 \% \\ 40 \% \sim 60 \% & =95 \% \sim 100 \%\end{array}$

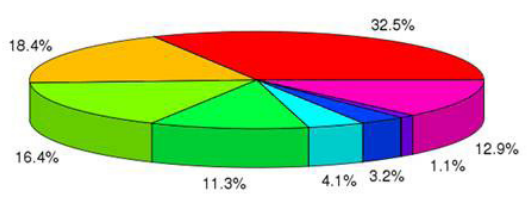

- Vitis vinifera Ricinus communis Populus trichocarpa - Glycine max Medicago truncatula Arabidopsis thaliana other

Figure 2. Characteristics of the homology search of assembled loquat unigenes against the $\mathrm{Nr}$ database. (A) E-value distribution of each loquat unigene with a cut-off E-value of $1.0 \mathrm{E}^{-5}$. (B) Similarity distribution of annotation unigenes. (C) Species distribution of all homologous unigenes with an E-value of at least 1.0E-5. 
Based on $\mathrm{Nr}$ annotation, $\mathrm{GO}$ analysis was used to classify the functions of the predicted loquat genes. The unigenes $(34,200)$ were categorized into three main categories, including biological process $(143,799)$, cellular component $(90,020)$, and molecular function $(39,826)$ (Figure 3). In the biological process domain, unigenes involved in cellular process and metabolic process were dominant, and there was also a high-percentage of unigenes in the response to stimulus term and biological regulation term. In the cellular component domain, cell and cell part were the highly represented terms, followed by organelle term and membrane term. Binding and catalytic activity were the principal terms for molecular function.

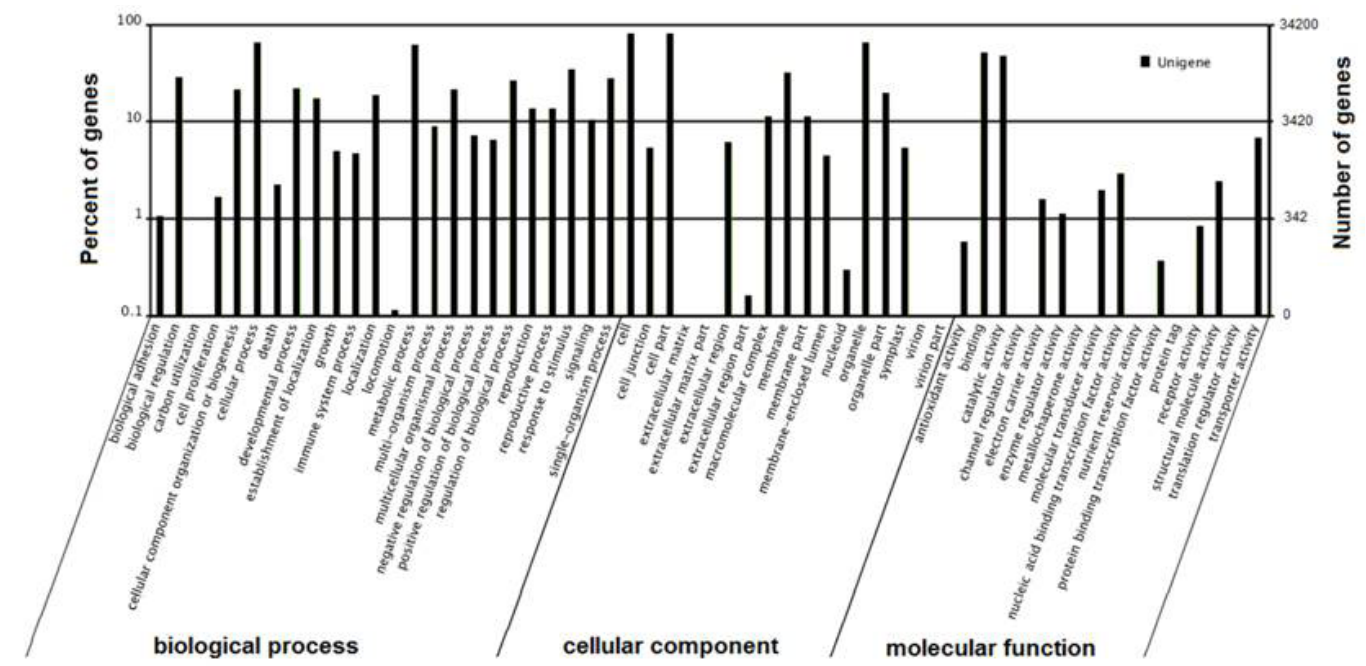

Figure 3. Functional annotation of loquat transcripts based on gene ontology categorization. A total of 34,200 unigenes were categorized into three main categories, including biological process $(143,799)$, cellular component $(90,020)$, and molecular function $(39,826)$. The left y-axis represents the percentage of a specific category of genes in the main category. The right $y$-axis represents the number of genes in a category.

To further predict and classify the unigenes, 14,538 unigenes were annotated by searching against the COG database. COG annotated putative proteins were functionally classified into at least 25 categories, such as cellular structure, biochemistry metabolism, molecular processing and signal transduction (Figure 4). The cluster for general function prediction represented the largest group $(4622 ; 15 \%)$, followed by transcription $(3181 ; 10.32 \%)$, replication, recombination, and repair $(2422 ; 7.86 \%)$, posttranslational modification, protein turnover and chaperones (2360; $7.66 \%)$, signal transduction mechanisms $(2060 ; 6.69 \%)$, translation, ribosomal structure, and biogenesis $(1969 ; 6.42 \%)$, carbohydrate transport and metabolism $(1889 ; 6.13 \%)$, and nuclear structure $(3 ; 00.1 \%)$ and extracellular structure $(16 ; 0.05 \%)$ represented the smallest groups (Figure 4$)$.

\section{Exploration of differentially expressed genes in response to low temperature stress}

To identify the effects of low temperature stress on the transcript levels of loquat unigenes, differentially expressed genes between samples from two treatments were identified using an algorithm developed by Audic and Claverie (1997). Two samples of 10 fruit spurs 
each were sampled from the crown of young fruit, of which 10 were placed in an artificial climate box at a processing low temperature stress of $3^{\circ} \mathrm{C}$, and another 10 were placed at a processing low temperature stress of $6^{\circ} \mathrm{C}$, for $12 \mathrm{~h}$. A total of 57,792 significantly altered unigenes were detected and mapped to 4017 genes, with 1619 genes upregulated and 2398 genes downregulated (Figures 5 and 6).

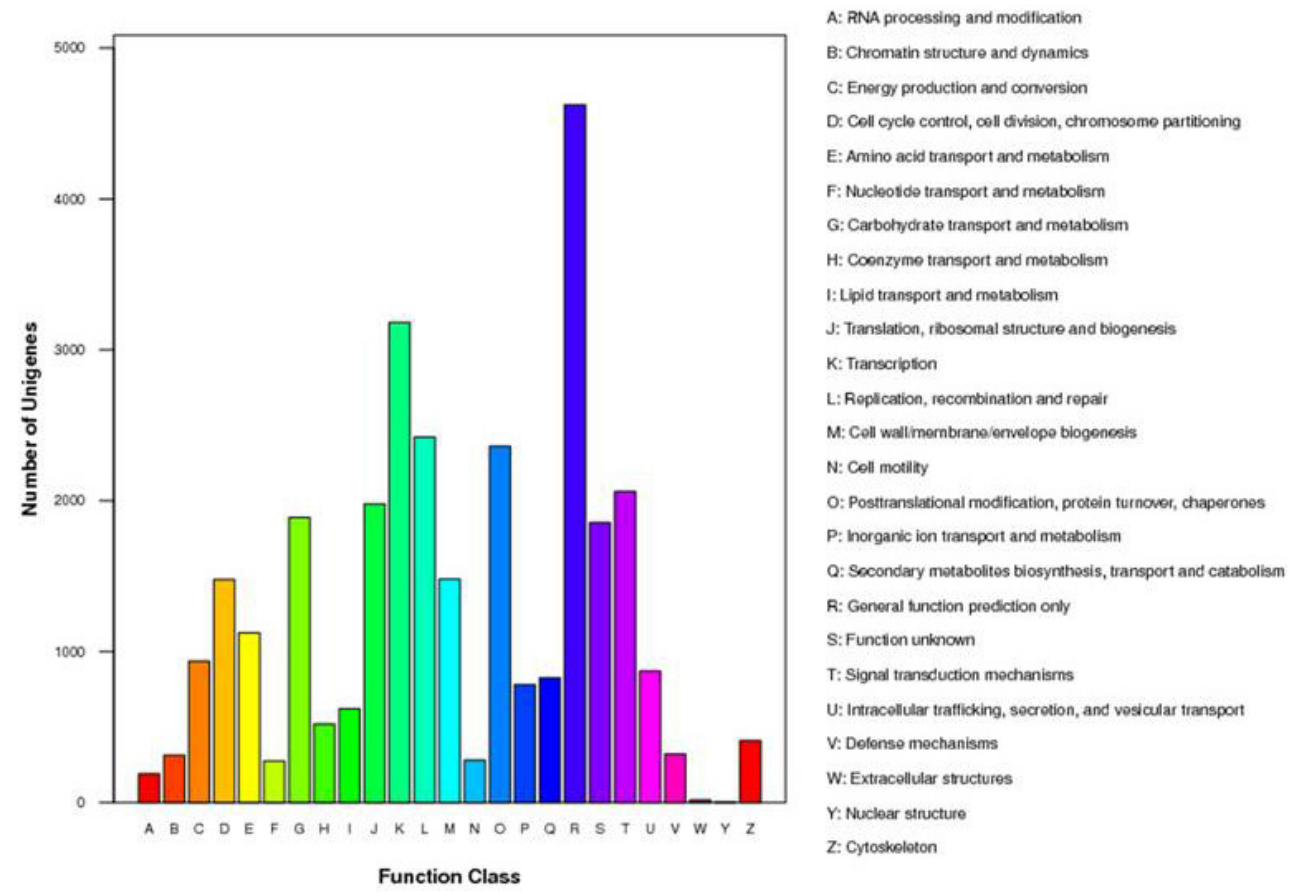

Figure 4. Functional annotation of loquat transcripts based on the clusters of orthologous groups dataset. A total of 14,538 unigenes were annotated and classified into at least 25 categories.

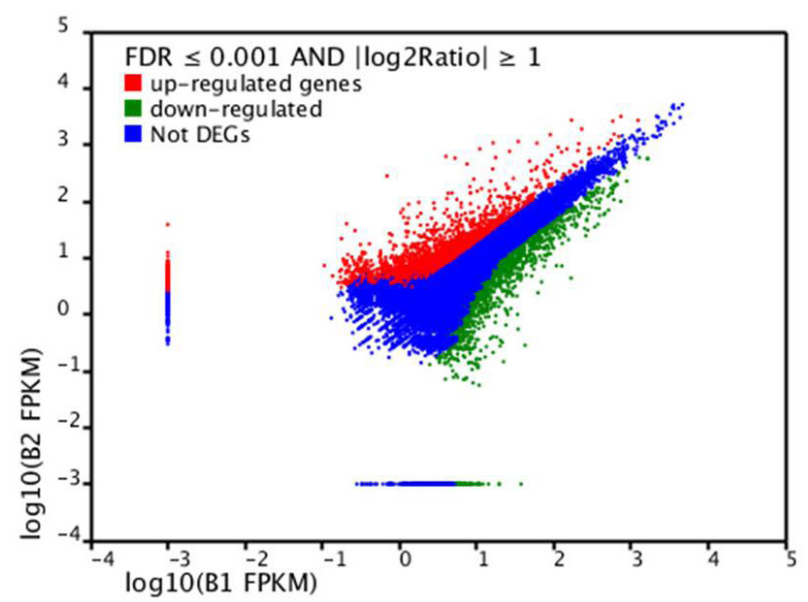

Figure 5. Difference in the expression of genes in loquat fruit maintained at $3^{\circ}$ and $6^{\circ} \mathrm{C}$ for $12 \mathrm{~h}$. 


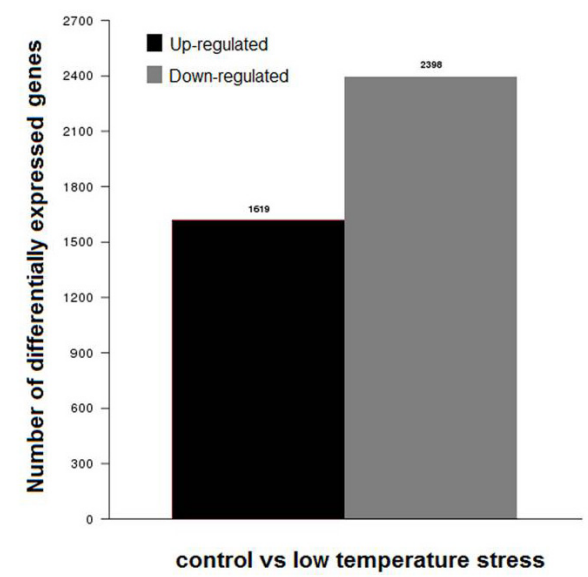

Figure 6. Changes in gene expression in loquat fruit under low temperature stress. The numbers of genes responding to low temperature stress are summarized above the bars.

\section{Functional annotation of differentially expressed genes}

We mapped all differentially expressed unigenes to the reference canonical pathways in the KEGG database, which is an alternative approach to categorize gene functions with an emphasis on biological pathways. In total, 113 pathways were enriched by a total of 2007 unigenes. The most representative pathways were metabolic pathways, biosynthesis of secondary metabolites, endocytosis, glycerophospholipid metabolism, plant hormone signal transduction, ether lipid metabolism, starch and sucrose metabolism, and plant-pathogen interaction. Notably, unigenes involved in the brassinosteroid biosynthesis and phosphatidylinositol signaling systems were upregulated. A total of 6 upregulated differentially expressed unigenes were found to participate in regulating brassinosteroid biosynthesis (Figure 7) and $P L C$ was upregulated in the phosphatidylinositol signaling system (Figure 8).

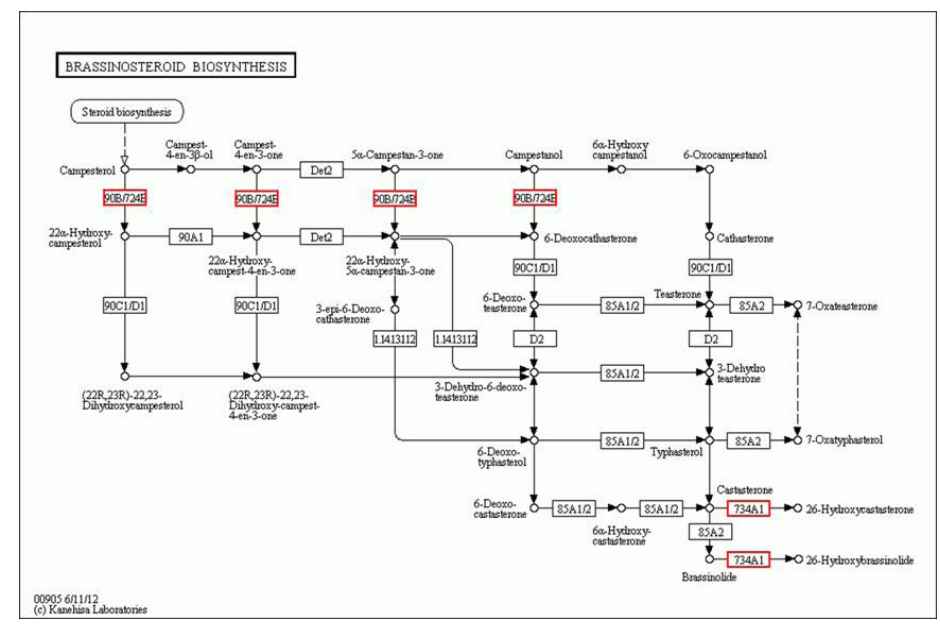

Figure 7. Putative brassinosteroid biosynthesis pathway in loquat generated using Kyoto encyclopedia of genes and genomes analysis. The numbers within the small boxes are enzyme codes. The genes boxed in red represent upregulated genes. 


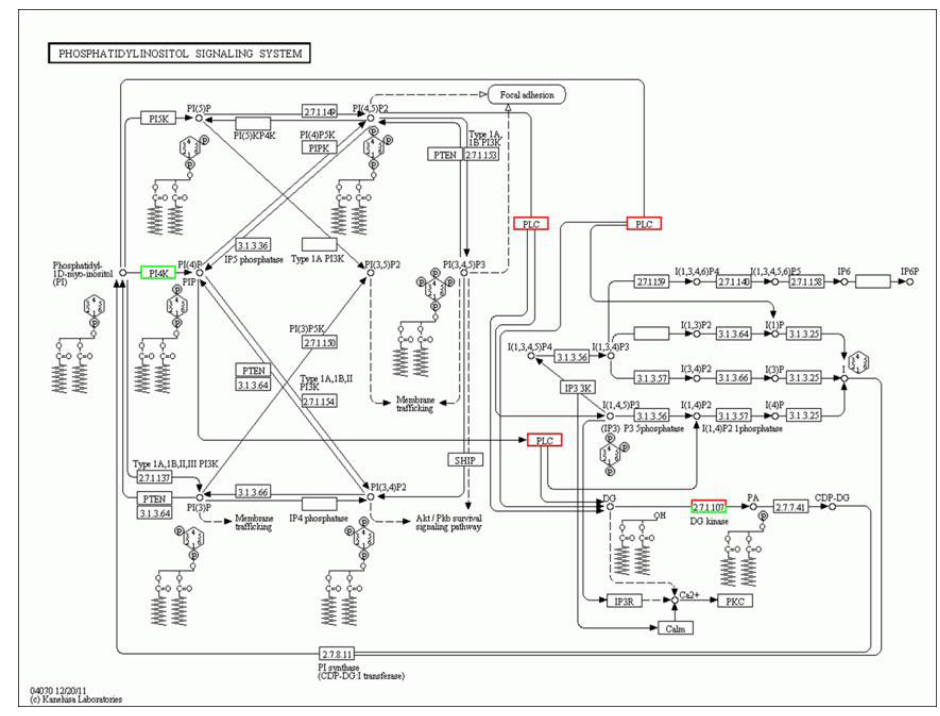

Figure 8. Putative phosphatidylinositol signaling pathway in loquat generated using Kyoto encyclopedia of genes and genomes analysis. PLC show in red is upregulated and downregulated genes are represented in green.

\section{Gene expression analysis under low temperature stress}

Two important genes regulating brassinolide biosynthesis are 25620 and CL8135. Gene 25620 plays a key role in the early C-6 oxidation pathway and CL8135 plays a key role in the late C-6 oxidation pathway. The expression patterns of these two genes are shown in Figure 9. The results show that the expression of gene 25620 was 2.9 times higher in samples maintained at $6^{\circ} \mathrm{C}$ compared to the control samples and 1277 times higher in samples from $3^{\circ} \mathrm{C}$ compared to the control. There was no difference in the expression of gene CL8135 in samples from $6^{\circ} \mathrm{C}$ and the control samples, but expression was 6.1 times higher in samples maintained at $3^{\circ} \mathrm{C}$ compared to the control samples. The results indicate that low temperature stress could induce expression of these two genes.

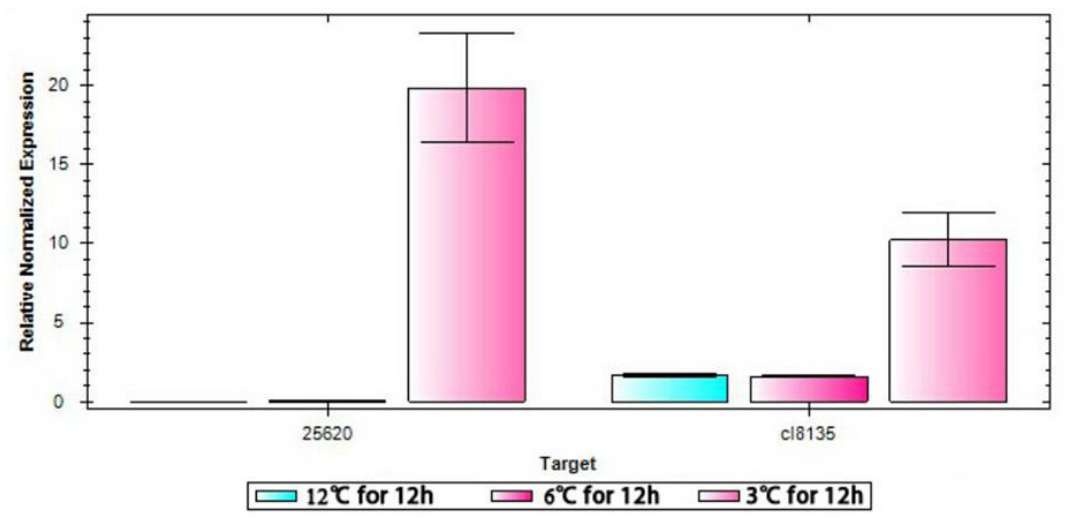

Figure 9. Expression patterns of the 25620 and CL 8135 genes in loquat fruit maintained under two low temperature treatments $\left(3^{\circ}\right.$ and $\left.6^{\circ} \mathrm{C}\right)$ and a control $\left(12^{\circ} \mathrm{C}\right)$ for $12 \mathrm{~h}$. 


\section{DISCUSSION}

The transcriptome is the complete set and quantity of transcripts in a tissue at a specific developmental stage or under a physiological condition. Based on transcriptomic information, an understanding of many biological processes is now possible, such as gene expression (Torres et al., 2008), gene expression profiles after experimental treatments or infection (Hegedus et al., 2009), gene regulation, discovery of tissue biomarkers, gene discovery, gene content, and isolation of conserved ortholog genes for phylogenomic purposes (Hughes et al., 2006), among others. Therefore, by taking advantage of next generation sequencing technology, transcriptome analysis provides invaluable genomic information for organisms. The Illumina sequencing technology has arisen as a powerful tool for sequencing transcriptomes, and many studies have utilized this technology for de novo assembly of non-model organisms' transcriptomes (Wang et al., 2010; Garg et al., 2011). However, transcriptomic information for loquat fruits is still very limited to date. Loquat, originates from Southwestern China (Zhang et al., 1990) and is now widely cultivated in many regions, including the subtropical regions of southern China, Japan, northern India, Israel, and the Mediterranean area. As one of the most important crop plants, loquat contains nearly all the essential nutrients, such as proteins, carbohydrates, cellulose, pectin, minerals, and carotenoids (Shaw, 1980).

In the present study, through de novo assembly of the transcriptome using Illumina pyrosequencing for a non-model plant, a large number of expressed genes were identified and analyzed for loquat. We generated more than twenty million sequence reads for loquat and a total of 45,739 unigenes were annotated by several complementary approaches. The annotations provide a valuable resource for investigating specific processes, functions, and pathways during loquat research. Our results indicate that Illumina sequencing technology is an efficient, inexpensive, and reliable platform for transcriptomic analysis for non-model organisms, and these findings provide a substantial contribution to loquat genome research.

Low temperature stress adversely affects plant growth and crop production and plants have developed a system to adapt to or resist unfavorable environments. Hundreds of genes have been identified in these processes, which participate in signal transduction, stress perception, and downstream responses (Kreps et al., 2002; Xiong et al., 2002; Zhu, 2002; Shinozaki et al., 2003). The purpose of this study was to identify genes that may be involved in the low temperature stress response of loquat. Using transcriptome analysis, 4017 genes (1619 upregulated and 2398 downregulated) were identified, furthering our understanding of the molecular mechanisms of the cold stress response in loquat.

Previous studies have demonstrated that many plant hormones are involved in coordinating the growth of loquat under different stresses. MeJA treatment can effectively inhibit chilling injury and inhibit anthracnose rot caused by Colletotrichum acutatum spores in postharvest loquat fruit (Cao et al., 2008, 2010), and acetylsalicylic acid, another plant hormone, was found to relieve chilling injury in loquat (Cai et al., 2006b). Thus, plant hormones have an important role in low temperature tolerance in loquat fruit. The functional analysis during the current study provided an overall picture of the loquat response to cold stress. In our experiment, 6 differentially expressed unigenes were upregulated, which participate in regulating brassinosteroid biosynthesis. Brassinosteroids (BRs), plant-specific steroid hormones, affect many aspects of plant growth and development. BRs have also been shown to protect plants from various environmental stresses. For instance, BRs were reported to promote cold stress 
tolerance in Arabidopsis seedlings (Divi and Krishna, 2010). Thus, our result suggests that BRs may be involved in low temperature stress in loquat fruit as well.

During the current study, we also observed that $P L C$ was upregulated in the phosphatidylinositol signaling system. PLC is a very important component in phosphatidylinositol signal transduction, which produces $\mathrm{PKC}$ and $\mathrm{Ca}^{2+}$ that link extracellular signals to intracellular events and responses to various abiotic stresses. Hydrolysis of inositol phospholipids by PLC is initiated by either receptor stimulation or opening of $\mathrm{Ca}^{2+}$ channels. Hirayama et al. (1995) found that AtPLC1 may be involved in the signal-transduction pathways of responses to environmental stresses and that an increase in the level of AtPLC1 may amplify the signal in a manner that contributes to the adaptation of the plant to these stresses (Hirayama et al., 1995). Expression of RD29A and COR47, the osmotic stress induction of the stress-responsive genes, was downregulated when PI-PLC activity was inhibited. We speculate that PLC may also participate in the low temperature response of loquat fruit.

In conclusion, genomic information for loquat is very limited and the current study is the first to provide the de novo assembled loquat transcriptome and to identify differentially expressed genes in loquat using Illumina sequencing technology. Here, we present the high-quality transcriptome sequence of loquat without the use of a reference genome. Annotation of the transcriptome provides greater insight into the gene content and functional categories in loquat, providing a significant contribution towards development of genomic resources for loquat and accelerating future genetic or genomic studies and breeding programs. Analysis of gene expression profiles under low temperature stress also demonstrates the feasibility of Illumina sequencing for functional studies of genes involved in low temperature stress. These findings will certainly improve our understanding of the molecular mechanisms of low temperature response in loquat.

\section{Conflicts of interest}

The authors declare no conflict of interest.

\section{ACKNOWLEDGMENTS}

Research supported by the Agriculture Science Technology Achievement Transformation Fund of China (\#2010GB2F000408) and the Fund of the Sichuan Provincial Education Department of China (\#2009ZA071). The authors thank BGI Tech Solutions Co. Ltd. for their excellent technical support.

\section{REFERENCES}

Audic S and Claverie JM (1997). The significance of digital gene expression profiles. Genome Res. 7: 986-995.

Altschul SF, Madden TL, Schaffer AA, Zhang J, et al. (1997). Gapped BLAST and PSI-BLAST: a new generation of protein database search programs. Nucleic Acids Res. 25: 3389-3402.

Cai C, Chen KS, Xu WP, Zhang WS, et al. (2006a). Effect of 1-MCP on postharvest quality of loquat fruit. Postharvest Biol. Technol. 40: 155-162.

Cai C, Li X and Chen KS (2006b). Acetylsalicylic acid alleviates chilling injury of postharvest loquat (Eriobotrya japonica Lindl.) fruit. Eur. Food Res. Technol. 223: 533-539.

Cai C, Xu CJ, Li X, Ferguson I, et al. (2006c). Accumulation of lignin in relation to change in activities of lignification enzymes in loquat fruit flesh after harvest. Postharvest Biol. Technol. 40: 163-169.

Cao SF, Zheng YH, Yang ZF, Tang SS, et al. (2008). Effect of methyl jasmonate on the inhibition of Colletotrichum acutatum infection in loquat fruit and the possible mechanisms. Postharvest Biol. Technol. 49: 301-307. 
Cao SF, Zheng YH, Wang KT, Rui HJ, et al. (2010). Effect of methyl jasmonate on cell wall modification of loquat fruit in relation to chilling injury after harvest. Food Chem. 118: 641-647.

Campbell C and Malo SE (1986). IFAS fact sheets 5. The Loquat. University of Florida, Gainsville.

Chen GG and Cai RB (1988). Biology and control of the pathogens of grey leaf spot of loquat. Acta Agriculturae Universitatis Zhejiangensis 14: 312-317.

Conesa A, Gotz S, Garcia-Gomez JM, Terol J, et al. (2005). Blast2GO: a universal tool for annotation, visualization and analysis in functional genomics research. Bioinformatics 21: 3674-3676.

Ding CK, Chachin K, Ueda Y and Mochioka R (1998). Changes in polyphenol concentrations and polyphenol oxidase activity of loquat (Eriobotrya japonica Lindl.) fruits in relation to browning. J. Jpn. Soc. Hort. Sci. 67: 360-366.

Ding CK, Chachin K, Ueda Y, Imahori Y, et al. (2002). Modified atmosphere packaging maintains postharvest quality of loquat fruit. Postharvest Biol. Technol. 24: 341-348.

Divi UK and Krishna P (2010). Overexpression of the brassinosteroid biosynthetic gene AtDWF4 in Arabidopsis seeds overcomes abscisic acid-induced inhibition of germination and increases cold tolerance in transgenic seedlings. $J$. Plant Growth Reg. 29: 385-393.

Garg R, Sahoo A, Tyagi AK and Jain M (2010). Validation of internal control genes for quantitative gene expression studies in chickpea (Cicer arietinum L.). Biochem. Biophys. Res. Commun. 396: 283-288.

Garg R, Patel RK, Tyagi AK and Jain M (2011). De novo assembly of chickpea transcriptome using short reads for gene discovery and marker identification. DNA Res. 18: 53-63.

Grabherr MG, Haas BJ, Yassour M, Levin JZ, et al. (2011). Full length transcriptome assembly from RNA-Seq data without a reference genome. Nat. Biotechnol. 29: 644-652.

Hegedus Z, Zakrzewska A, Agoston VC, Ordas A, et al. (2009). Deep sequencing of the zebrafish transcriptome response to mycobacterium infection. Mol. Immunol. 46: 2918-2930.

Hirayama T, Ohto C, Mizoguchi T and Shinozaki K (1995). A gene encoding a phosphatidylinositol-specific phospholipase C is induced by dehydration and salt stress in Arabidopsis thaliana. Proc. Natl. Acad. Sci. U. S. A. 92: 3903-3907.

Huang W and Marth G (2008). EagleView: a genome assembly viewer for next generation sequencing technologies. Genome Res. 18: 1538-1543.

Hughes J, Longhorn SJ, Papadopoulou A, Theodorides K, et al. (2006). Dense taxonomic EST sampling and its applications for molecular systematics of the Coleoptera (beetles). Mol. Biol. Evol. 23: 268-278.

Jiang Y, Joyce DC and Terry LA (2001). 1-Methylcyclopropene treatment affects strawberry fruit decay. Postharvest Biol. Technol. 23: 227-232.

Kreps JA, Wu Y, Chang HS, Zhu T, et al. (2002). Transcriptome changes for Arabidopsis in response to salt, osmotic, and cold stress. Plant Physiol. 130: 2129-2141.

Krost C, Petersen R and Schmidt ER (2012). The transcriptomes of columnar and standard type apple trees (Malus x domestica) - A comparative study. Gene 498: 223-230.

Lin SQ (2007). World loquat production and research with special reference to China. Acta. Hortic. 750: 37-44.

Lin S, Sharpe RH and Janick J (1999). Loquat: botany and horticulture. Hort. Rev. 23: 233-276.

Mortazavi A, Williams BA, McCue K, Schaeffer L, et al. (2008). Mapping and quantifying mammalian transcriptomes by RNA-Seq. Nat. Methods 5: 621-628.

Morton JF (1987). Loquat. In: Fruits of warm climates. Creative Resource Systems, Winterville, 103-108.

Rosenkranz R, Borodina T, Lehrach H and Himmelbauer H (2008). Characterizing the mouse ES cell transcriptome with Illumina sequencing. Genomics 92: 187-194.

Shaw PE (1980). Loquat. In: Tropical and subtropical fruits: composition, properties and uses (Nagy S and Shaw PE, eds.). AVI, Westport, 480-481.

Shinozaki K, Yamaguchi-Shinozaki K and Seki M (2003). Regulatory network of gene expression in the drought and cold stress responses. Curr. Opin. Plant Biol. 6: 410-417.

Torres TT, Metta M, Ottenwalder B and Schlotterer C (2008). Gene expression profiling by massively parallel sequencing. Genome Res. 18: 172-177.

Varshney RK, Graner A and Sorrells ME (2005). Genomics-assisted breeding for crop improvement. Trends Plant Sci. 10: 621-630.

Varshney RK, Hoisington DA and Tyagi AK (2006). Advances in cereal genomics and applications in crop breeding. Trends Biotechnol. 24: 490-499.

Wang XW, Luan JB, Li JM, Bao YY, et al. (2010). De novo characterization of a whitefly transcriptome and analysis of its gene expression during development. BMC Genomics 11: 400 .

Xiong L, Schumaker KS and Zhu JK (2002). Cell signaling during cold, drought, and salt stress. Plant Cell 14: S165-183.

Zhang HZ, Peng SA, Cai LH and Fang DQ (1990). The germplasm resources of the genus Eriobotrya with special reference on the origin of E. japonica Lindl. Acta Hortic. Sin. 17: 5-12.

Zhang HZ, Peng SA, Cai LH and Fang DQ (1993). The germplasm resources of the genus Eriobotrya with special 
reference on the origin of E. japonica Lindl. Plant Breed. Abstr. 63: 772.

Zheng YH, Li SY and Xi YF (2000). Changes of cell wall substances in relation to flesh woodiness in cold-stored loquat fruits. Acta Phytophysiol. Sin. 26: 306-310.

Zhu JK (2002). Salt and drought stress signal transduction in plants. Annu. Rev. Plant Biol. 53: 247-273. 